\title{
Unexpected substituent effects in aryl-aryl Negishi cross-coupling reactions rationalized by DFT and natural charges
}

\author{
Felix Witte*1, Sina P. Zucker ${ }^{2}$, Beate Paulus ${ }^{1}$, and C. Christoph Tzschucke ${ }^{2}$
}

${ }^{1}$ Freie Universität Berlin, Institute of Chemistry and Biochemistry, Arnimallee 22, 14195 Berlin, Germany

${ }^{2}$ Freie Universität Berlin, Institute of Chemistry and Biochemistry, Arnimallee 22, 14195 Berlin, Germany

E-mail: jf.witte@fu-berlin.de 


\section{Supporting Information}

\section{Contents}

1 General information

2 Synthetic procedures and spectral characterization

2.1 Tris(dibenzylideneacetone)dipalladium(0) chloroform adduct, $\mathrm{Pd}_{2} \mathrm{dba}_{3} \cdot \mathrm{CHCl}_{3}$ (palladium precursor) $\mathrm{S} 4$

2.2 cis-(tmeda)Pd(Ar)l complexes . . . . . . . . . . . . . . . . . . . . . . . S4

2.3 cis-(dppe) $\mathrm{Pd}(\mathrm{Ar}) \mathrm{I}$ complexes . . . . . . . . . . . . . . . . . . . . . . . . . S S7

3 Competition experiments $\quad$ S11

4 Additional computational results $\quad$ S14 


\section{General information}

All air and moisture sensitive reactions and experiments were carried out using oven-dried glassware under an argon atmosphere. Reactions were set up either in glove box using glass vials with a Teflon-lined screw cap and heated on an aluminum well plate or by using Schlenk techniques and heated in a pre-heated oil bath using a stirrer and heater with temperature control.

Chemicals were purchased from commercial suppliers and used as received unless otherwise noted. Toluene, $\mathrm{THF}$, pentane, $\mathrm{Et}_{2} \mathrm{O}$, dioxane and deuterated solvents (THF, toluene, benzene) were dried and distilled over sodium/benzophenone and degassed using freeze-pump-thaw technique. MeCN was dried and distilled from $\mathrm{CaH}_{2}$. Dry $\mathrm{CH}_{2} \mathrm{Cl}_{2}$ was purchased from Sigma-Aldrich and dried via Solvent Purification System MB-SPS 800 from MBraun. Dry DMF, $\mathrm{MeOH}$ and $\mathrm{EtOH}$ were purchased from Acros in AcroSeal bottles.

Column chromatography was performed on Merck Silica Gel 60 (230-400 mesh) or Acros Aluminium Oxide, basic, Brockmann I, 50-200 $\mu \mathrm{m}$. Hexane and ethyl acetate were previously distilled using rotary evaporation.

Thin layer chromatography was performed on Merck TLC Silica gel 60 F 254. Compounds were detected using an UV/Vis lamp (254 $\mathrm{nm})$.

${ }^{1} \mathrm{H},{ }^{13} \mathrm{C},{ }^{19} \mathrm{~F}$ and ${ }^{31} \mathrm{P}$ NMR spectra were recorded on Jeol ECX 400 or Bruker Avance500 or Joel ECX 400 at room temperature unless otherwise noted. Data were processed by MestReNova Version: 7.1.2-10008. Chemical shifts $(\delta)$ are reported in parts per million (ppm) and referenced to residual solvent peak $\left(\mathrm{CDCl}_{3}:{ }^{1} \mathrm{H} \delta=7.26 \mathrm{ppm}\right.$, ${ }^{13} \mathrm{C} \delta=77.16 \mathrm{ppm} ; \mathrm{C}_{6} \mathrm{D}_{6}:{ }^{1} \mathrm{H} \delta=7.16 \mathrm{ppm},{ }^{13} \mathrm{C} \delta=128.06 \mathrm{ppm} ;{ }^{31} \mathrm{P}$ and ${ }^{19} \mathrm{~F}$ : frequency calibrated log with $\pm 1 \mathrm{ppm}$ deviation). Multiplicities are given as follows: $\mathrm{s}=$ singlet, $\mathrm{d}=$ doublet, $\mathrm{t}=$ triplet, $\mathrm{q}=$ quartet and $\mathrm{m}$ $=$ multiplet. Coupling constants $(\mathrm{J})$ are reported in $\operatorname{Hertz}(\mathrm{Hz})$.

High resolution ESI-MS spectra were recorded on an Agilent 6210 ESI-TOF, Agilent Technologies. The applied charge is reported as positive $(+)$ or negative $(-)$. The spray charge was set to $4 \mathrm{kV}$. Data are reported in mass to charge $(\mathrm{m} / \mathrm{z})$.

IR spectra were recorded on a Jasco FT/IR-4100 FT-IR with a ZnSe optical window. The absorption bands are given in wave numbers $\left(\mathrm{cm}^{-1}\right)$, intensities are reported as follows: $\mathrm{s}=$ strong, $\mathrm{m}=$ medium, $\mathrm{w}=$ weak. Kinetic measurements by in situ IR were performed with Mettler Toledo ReactIR 15.

Melting points (m.p.) were determined on a BÜCHI 510 melting point apparatus and are uncorrected.

Elemental analyses for contents of carbon, nitrogen, hydrogen, and sulfur were performed on an elemental analyzer VARIO EL, Elementar, and results are reported in percentage (\%).

Quantitative gas chromatography measurements were performed on a Varian CP-3800 equipped with a Varian CP-8400 autosampler, a FID detector and a fused silica capillary column (CP-Sil 8 CB or F\&W VF-5ms, 5\% phenyl and $95 \%$ dimethylsiloxane phase, $30 \mathrm{~m} \times 0.25 \mathrm{~mm}$ ID $\times 0.25 \mu \mathrm{m}$ film). Quantifications of analytes were done using the internal standard approach from 10 points calibrations. All calibration and sample solutions were measured three times. The injection volume was $1 \mu \mathrm{mol}$ unless otherwise noted.

Qualitative gas chromatography measurements were performed on a 7820A gas chromatograph equipped with a fused silica capillary column (F\&W VF-5ms, 5\% phenyl and 95\% dimethylsiloxane phase, $30 \mathrm{~m} \times 0.25 \mathrm{~mm}$ ID $\times 0.25 \mu \mathrm{m}$ film), 5977E MSD (single quadrupole) detector from Agilent Technologies and Varian CP-8410 autosampler. Nitrogen was employed as carrier and make-up gas. The data were processed with Varian Star Chromatography Workstation Version 6.41. 


\section{Synthetic procedures and spectral characterization}

\subsection{Tris(dibenzylideneacetone)dipalladium(0) chloroform adduct, $\mathrm{Pd}_{2} \mathrm{dba}_{3} \cdot \mathrm{CHCl}_{3}$ (palladium precursor)}<smiles>O=C(/C=C/c1ccccc1)/C=C/c1ccccc1</smiles>

According to the reported procedure, ${ }^{1} 500 \mathrm{~mL}$ single necked-flask was charged with $\mathrm{PdCl} 2(2.68 \mathrm{~g}, 15.1 \mathrm{mmol})$, dibenzylidineacetone $(12.3 \mathrm{~g}, 52.4 \mathrm{mmol}), \mathrm{NaOAc}(10.5 \mathrm{~g}, 128 \mathrm{mmol})$ and $\mathrm{MeOH}(300 \mathrm{~mL})$. After the resulting mixture has been stirred at $40{ }^{\circ} \mathrm{C}$ for $3 \mathrm{~h}$, the solid was filtered by suction filtration using a glass frit (D3). The solid was first washed with water until the filtrate was neutral, then with $\mathrm{MeOH}$ (twice). The purple solid was dissolved in $\mathrm{CDCl}_{3}(1.20 \mathrm{~L})$ and the deep purple solution concentrated $(\approx 250 \mathrm{~mL})$. Acetone $(800 \mathrm{~mL})$ was added and the resulting mixture was kept at $-25{ }^{\circ} \mathrm{C}$ over night. The formed solid was again filtered by suction filtration using a glass frit (D3) and dried under vacuum providing the title compound (15.4 $\mathrm{g}, 99 \%)$ as crystalline, dark purple solid, which was used without further purification.

\section{2 cis-(tmeda) $\mathrm{Pd}(\mathrm{Ar}) \mathrm{I}$ complexes}

General procedure:

An oven-dried Schlenk flask was charged with $\mathrm{Pd}_{2} \mathrm{dba}_{3} \cdot \mathrm{CHCl}_{3}$ (1 equiv.) and aryl iodide (2.5-3.0 equiv.), sealed with rubber septum and set under an atmosphere of argon by cycled evacuation and backfilling with argon. Dry toluene $(0.05 \mathrm{M})$ and TMEDA (2.5 equiv.) were syringed to the reaction flask and the resulting deep purple mixture was stirred at $50{ }^{\circ} \mathrm{C}$ for 30-60 min, during which the purple color disappeared. After cooling to room temperature, the mixture was filtered through a pad of celite and the filter washed with DCM. The combined filtrates were concentrated under vacuum, $\mathrm{Et}_{2} \mathrm{O}(50.0 \mathrm{~mL})$ was added and the resulting solution kept at $-25{ }^{\circ} \mathrm{C}$ for approximately $3 \mathrm{~h}$. The supernatant liquid was decanted, the solid washed with additional $\mathrm{Et}_{2} \mathrm{O}$ until the liquid phase was colorless and the solid dried in vacuum.

\section{cis-(tmeda) $\mathrm{Pd}\left(p-\mathrm{MeOC}_{6} \mathrm{H}_{4}\right) I^{2}$ (pre-OMe)}<smiles>COc1ccc([Pb]2(I)O[N+]3(C)N(C)CCN32)cc1</smiles>

According to the general procedure, the reaction of $\mathrm{Pd}_{2} \mathrm{dba}_{3} \cdot \mathrm{CHCl}_{3}(1.03 \mathrm{~g}, 0.99 \mathrm{mmol})$, TMEDA $(0.38 \mathrm{~mL}, 2.54 \mathrm{mmol})$ and 4-bromoanisole $(0.67 \mathrm{~g}, 2.88 \mathrm{mmol})$ in toluene $(20.0 \mathrm{~mL})$ provided the complex $(0.80 \mathrm{~g}, 1.76 \mathrm{mmol}, 89 \%)$ as orange solid.

${ }^{1} \mathrm{H} \mathrm{NMR}\left(400 \mathrm{MHz}, \mathrm{CDCl}_{3}\right): \delta 7.08(\mathrm{~d}, J=8.7 \mathrm{~Hz}, 2 \mathrm{H}), 6.60(\mathrm{~d}, J=8.7 \mathrm{~Hz}, 2 \mathrm{H}), 3.70(\mathrm{~s}, 3 \mathrm{H}), 2.71-2.67(\mathrm{~m}, 2 \mathrm{H})$, $2.65(\mathrm{~s}, 6 \mathrm{H}), 2.56-2.51(\mathrm{~m}, 2 \mathrm{H}), 2.30(\mathrm{~s}, 6 \mathrm{H}) \mathrm{ppm}$.

${ }^{13} \mathrm{C}$ NMR $\left(101 \mathrm{MHz}, \mathrm{CDCl}_{3}\right): \delta 156.5,136.1,131.8,113.0,62.2,58.4,55.2,49.9,49.8 \mathrm{ppm}$.

IR $\left(\nu / \mathrm{cm}^{-1}\right)$ : $2976(\mathrm{w}), 2885(\mathrm{w}), 2834(\mathrm{w}), 2791(\mathrm{w}), 1576(\mathrm{w}), 1477(\mathrm{~m}), 1454(\mathrm{~m}), 1405(\mathrm{w}), 1268(\mathrm{~m}), 1226(\mathrm{~m})$, $1170(\mathrm{~m}), 1124(\mathrm{w}), 1095(\mathrm{w}), 1065(\mathrm{w}), 1047(\mathrm{w}), 1023(\mathrm{~m}), 1003(\mathrm{~m}), 957(\mathrm{~m}), 825(\mathrm{~m}), 802(\mathrm{~s}), 772(\mathrm{~m})$.

HRESI-MS $(\mathrm{MeOH},+, \mathrm{m} / z)$ : $[\mathrm{M}+\mathrm{Na}]^{+}$calcd for $\mathrm{C}_{13} \mathrm{H}_{23} \mathrm{IN}_{2} \mathrm{NaOPd}^{+}$, 478.9782; found, 478.9788. $[\mathrm{M}+\mathrm{K}]^{+}$calcd for $\mathrm{C}_{13} \mathrm{H}_{23} \mathrm{IKN}_{2} \mathrm{OPd}^{+}$, 494.9521; found, 494.9523.

Elemental analysis (\%): calcd for $\mathrm{C}_{13} \mathrm{H}_{23} \mathrm{IN}_{2} \mathrm{OPd}, \mathrm{C} 34.2, \mathrm{H}$ 5.08, N 6.13; found, C 33.8, H 5.11, N 5.90.

M.p.: $155{ }^{\circ} \mathrm{C}$ (decomp.) 


\section{cis-(tmeda) $\mathrm{Pd}\left(p-\mathrm{MeC}_{6} \mathrm{H}_{4}\right) I^{2}$ (pre-Me)}<smiles>Cc1ccc([Pb]2(I)N(C)CCN2C)cc1</smiles>

According to the general procedure, the reaction of $\mathrm{Pd}_{2} \mathrm{dba}_{3} \cdot \mathrm{CHCl}_{3}(3.01 \mathrm{~g}, 2.91 \mathrm{mmol})$, TMEDA $(1.13 \mathrm{~mL}, 7.54 \mathrm{mmol})$ and 4-iodotoluene $(1.83 \mathrm{~g}, 8.41 \mathrm{mmol})$ in toluene $(40.0 \mathrm{~mL})$ provided the complex $(2.18 \mathrm{~g}, 4.95 \mathrm{mmol}, 85 \%)$ as orange solid.

${ }^{1} \mathrm{H} \mathrm{NMR}\left(400 \mathrm{MHz}, \mathrm{CDCl}_{3}\right): \delta 7.09(\mathrm{~d}, J=8.1 \mathrm{~Hz}, 2 \mathrm{H}), 6.75(\mathrm{~d}, J=7.5 \mathrm{~Hz}, 2 \mathrm{H}), 2.73-2.67(\mathrm{~m}, 2 \mathrm{H}), 2.65(\mathrm{~s}, 6 \mathrm{H})$, 2.56-2.51 (m, $2 \mathrm{H}), 2.31(\mathrm{~s}, 6 \mathrm{H}), 2.21(\mathrm{~s}, 3 \mathrm{H}) \mathrm{ppm}$.

${ }^{13} \mathrm{C}$ NMR $\left(101 \mathrm{MHz}, \mathrm{CDCl}_{3}\right): \delta 139.6,139.5,136.1,131.7,127.7,62.2,58.3,50.0,49.8,20.7 \mathrm{ppm}$.

IR $\left(\nu / \mathrm{cm}^{-1}\right): 2991(w), 2973(w), 2882(w), 2838(w), 2787(w), 1738(w), 1476(m), 1451(m), 1281(w), 1206(w)$, 1124 (w), 1097 (w), $1056(\mathrm{~m}), 1021(\mathrm{~m}), 1010(\mathrm{~m}), 957$ (m), 933 (w), 798 (s), $771(\mathrm{~m})$.

HRESI-MS $(\mathrm{MeOH},+, \mathrm{m} / \mathrm{z}):[\mathrm{M}+\mathrm{Na}]^{+}$calcd for $\mathrm{C}_{13} \mathrm{H}_{23} \mathrm{IN}_{2} \mathrm{NaPd}^{+}, 462.9833$; found, 462.9837. $[\mathrm{M}+\mathrm{K}]^{+}$calcd for $\mathrm{C}_{13} \mathrm{H}_{23} \mid \mathrm{KN}_{2} \mathrm{Pd}^{+}, 478.9572$; found, 478.9569 .

Elemental analysis (\%): calcd for $\mathrm{C}_{13} \mathrm{H}_{23} \mathrm{IN}_{2} \mathrm{Pd}, \mathrm{C} 35.5, \mathrm{H}$ 5.26, N 6.36; found, C 35.5, H 5.39, N 6.05.

M.p.: $165{ }^{\circ} \mathrm{C}$ (decomp.)

\section{cis-(tmeda) $\left.\mathrm{Pd}\left(\mathrm{C}_{6} \mathrm{H}_{5}\right)\right|^{3}$ (pre-H)}<smiles></smiles>

According to the general procedure, the reaction of $\mathrm{Pd}_{2} \mathrm{dba}_{3} \cdot \mathrm{CHCl}_{3}(3.02 \mathrm{~g}, 2.92 \mathrm{mmol})$, TMEDA $(1.13 \mathrm{~mL}, 7.54 \mathrm{mmol})$ and iodobenzene $(0.93 \mathrm{~mL}, 8.40 \mathrm{mmol})$ in toluene $(40.0 \mathrm{~mL})$ provided the complex $(2.23 \mathrm{mg}, 5.23 \mathrm{mmol}, 90 \%)$ as pale orange solid.

${ }^{1} \mathrm{H}$ NMR $\left(400 \mathrm{MHz}, \mathrm{CDCl}_{3}\right): \delta 7.24(\mathrm{dd}, J=8.0,1.2 \mathrm{~Hz}, 2 \mathrm{H}), 6.91(\mathrm{dd}, J=8.1,6.9 \mathrm{~Hz}, 2 \mathrm{H}), 6.84-6.74(\mathrm{~m}, 1 \mathrm{H})$, 2.74-2.70 (m, $2 \mathrm{H}), 2.67(\mathrm{~s}, 6 \mathrm{H}), 2.57-2.53(\mathrm{~m}, 2 \mathrm{H}), 2.32(\mathrm{~s}, 6 \mathrm{H}) \mathrm{ppm}$.

${ }^{13} \mathrm{C} \mathrm{NMR}\left(101 \mathrm{MHz}, \mathrm{CDCl}_{3}\right): \delta 144.7,136.6,126.7,122.8,62.3,58.4,50.0,49.9 \mathrm{ppm}$.

IR $\left(\nu / \mathrm{cm}^{-1}\right)$ : $3046(\mathrm{w}), 2992(\mathrm{w}), 2966(\mathrm{w}), 2885(\mathrm{w}), 2837(\mathrm{w}), 2789(\mathrm{w}), 1561(\mathrm{~m}), 1456(\mathrm{~m}), 1421(\mathrm{w}), 1285(\mathrm{~m})$, $1192(w), 1164(w), 1127(w), 1101(w), 1061(m), 1046(w), 1019(m), 991(w), 955(m), 933(w), 800(s), 770(m)$, $742(\mathrm{~s}), 699(\mathrm{~m}), 658(\mathrm{w})$.

HRESI-MS $(\mathrm{MeOH},+, \mathrm{m} / \mathrm{z}):[\mathrm{M}+\mathrm{Na}]^{+}$calcd for $\mathrm{C}_{12} \mathrm{H}_{21} \mathrm{IN}_{2} \mathrm{NaPd}^{+}$, 464.9416; found, 448.9676, $[\mathrm{M}+\mathrm{K}]^{+}$calcd for $\mathrm{C}_{12} \mathrm{H}_{21} I \mathrm{KN}_{2} \mathrm{Pd}^{+}, 464,9416$; found, 464.9499 .

Elemental analysis (\%): calcd for $\mathrm{C}_{12} \mathrm{H}_{21} \mathrm{IN}_{2} \mathrm{Pd}, \mathrm{C} 33.8, \mathrm{H}$ 4.96, N 6.57; found, C 33.9, H 5.03, N 6.63.

M.p.: $173^{\circ} \mathrm{C}$ (decomp.)<smiles>CC(C)[C@H](C)CC(C)(C)C</smiles>

According to the general procedure, the reaction of $\mathrm{Pd}_{2} \mathrm{dba}_{3} \cdot \mathrm{CHCl}_{3}(1.02 \mathrm{~g}, 0.99 \mathrm{mmol})$, TMEDA (0.38 mL, $2.54 \mathrm{mmol})$ and 4-fluoroiodobenzene $(0.33 \mathrm{~mL}, 2.86 \mathrm{mmol})$ in toluene $(20.0 \mathrm{~mL})$ provided the complex $(0.93 \mathrm{~g}, 1.97 \mathrm{mmol}, 99 \%)$ as pale orange solid. 
${ }^{1} \mathrm{H}$ NMR $\left(400 \mathrm{MHz}, \mathrm{CDCl}_{3}\right): \delta 7.15(\mathrm{dd}, J=8.6,6.3 \mathrm{~Hz}, 2 \mathrm{H}), 6.71(\mathrm{dd}, J=9.6,8.7 \mathrm{~Hz}, 2 \mathrm{H}), 2.72-2.68(\mathrm{~m}, 2 \mathrm{H})$, $2.66(\mathrm{~s}, 6 \mathrm{H}), 2.57-2.52(\mathrm{~m}, 2 \mathrm{H}), 2.29(\mathrm{~s}, 6 \mathrm{H}) \mathrm{ppm}$.

${ }^{13} \mathrm{C}$ NMR $\left(101 \mathrm{MHz} \mathrm{CDCl}_{3}\right): \delta 162.1,159.8,136.5,113.4,62.2,58.4,50.0,49.9 \mathrm{ppm}$.

${ }^{19} \mathrm{~F}$ NMR $\left(376 \mathrm{MHz} \mathrm{CDCl}_{3}\right): \delta-123.83(\mathrm{dt}, J=14.5,7.0 \mathrm{~Hz}) \mathrm{ppm}$.

IR $\left(\nu / \mathrm{cm}^{-1}\right): 2978(w), 2884(w), 2836(w), 2796(w), 1566(w), 1473(\mathrm{~m}), 1460(\mathrm{~m}), 1438(\mathrm{~m}), 1406(\mathrm{w}), 1375(\mathrm{w})$, $1248(\mathrm{w}), 1200$ (s), $1153(\mathrm{~m}), 1128$ (w), 1106 (w), 1081 (w), 1068 (w), 1052 (m), $1026(\mathrm{~m}), 1007$ (m), $959(\mathrm{~m}), 937$ $(\mathrm{w}), 815(\mathrm{~s}), 805(\mathrm{~s}), 775(\mathrm{~m})$.

HRESI-MS $(\mathrm{MeOH},+, \mathrm{m} / \mathrm{z})$ : $[\mathrm{M}+\mathrm{Na}]^{+}$calcd for $\mathrm{C}_{12} \mathrm{H}_{20} \mathrm{FIN}_{2} \mathrm{NaPd}^{+}, 466.9582$; found, 466.9590. [M $\left.+\mathrm{K}\right]^{+}$calcd for $\mathrm{C}_{12} \mathrm{H}_{20} \mathrm{FIKN}_{2} \mathrm{Pd}^{+}$, 482.9322; found, 482.9318.

Elemental analysis (\%): calcd for $\mathrm{C}_{12} \mathrm{H}_{20} \mathrm{FIN}_{2} \mathrm{Pd}, \mathrm{C} 32.4, \mathrm{H} \mathrm{4.53,} \mathrm{N} \mathrm{6.30;} \mathrm{found,} \mathrm{C} \mathrm{32.6,} \mathrm{H} \mathrm{4.56,} \mathrm{N} \mathrm{6.31.}$

M.p.: $145-147^{\circ} \mathrm{C}$ (decomp.)

\section{cis-(tmeda) $\left.\mathrm{Pd}\left(p-\mathrm{EtO}_{2} \mathrm{CC}_{6} \mathrm{H}_{4}\right)\right|^{5}$ (pre- $\left.\mathrm{CO}_{2} \mathrm{Et}\right)$}<smiles>CCOC(=O)c1ccc([Pb]2(I)N(C)CCN2C)cc1</smiles>

According to the general procedure, the reaction of $\mathrm{Pd}_{2} \mathrm{dba}_{3} \cdot \mathrm{CHCl}_{3}(1.02 \mathrm{~g}, 0.98 \mathrm{mmol})$, TMEDA $(0.38 \mathrm{~mL}, 2.54 \mathrm{mmol})$ and ethyl 4-iodobenzoate $(0.79 \mathrm{~g}, 2.87 \mathrm{mmol})$ in toluene $(20.0 \mathrm{~mL})$ provided the complex $(0.87 \mathrm{~g}, 1.75 \mathrm{mmol}, 88 \%)$ as pale yellow solid.

${ }^{1} \mathrm{H}$ NMR $\left(400 \mathrm{MHz} \mathrm{CDCl}_{3}\right): \delta 7.54(\mathrm{~d}, J=8.4 \mathrm{~Hz}, 2 \mathrm{H}), 7.39(\mathrm{~d}, J=8.4 \mathrm{~Hz}, 2 \mathrm{H}), 4.28(\mathrm{q}, J=7.1 \mathrm{~Hz}, 2 \mathrm{H}), 2.69-2.66$ $(\mathrm{m}, 2 \mathrm{H}), 2.65(\mathrm{~s}, 6 \mathrm{H}), 2.54-2.50(\mathrm{~m}, 2 \mathrm{H}), 2.28(\mathrm{~s}, 6 \mathrm{H}), 1.32(\mathrm{t}, J=7.1 \mathrm{~Hz}, 3 \mathrm{H}) \mathrm{ppm}$.

${ }^{13} \mathrm{C}$ NMR $\left(101 \mathrm{MHz} \mathrm{CDCl}_{3}\right): \delta 167.8,156.5,136.6,126.6,125.2,62.2,60.4,58.4,50.0,49.9,14.5 \mathrm{ppm}$.

IR $\left(\nu / \mathrm{cm}^{-1}\right)$ : $2989(\mathrm{w}), 2892(\mathrm{w}), 2870(\mathrm{w}), 2838(\mathrm{w}), 1689(\mathrm{~m}), 1570(\mathrm{~m}), 1469(\mathrm{~m}), 1385(\mathrm{w}), 1366(\mathrm{w}), 1303(\mathrm{w})$, $1277(\mathrm{~s}), 1264(\mathrm{~s}), 1175(\mathrm{~m}), 1104(\mathrm{~m}), 1090(\mathrm{~m}), 1056(\mathrm{w}), 1014(\mathrm{~m}), 956(\mathrm{~m}), 932(\mathrm{w}), 834(\mathrm{w}), 799(\mathrm{~s}), 761(\mathrm{~s}), 698$ (w).

HRESI-MS $(\mathrm{MeOH},+, \mathrm{m} / \mathrm{z})$ : $[\mathrm{M}-\mathrm{l}]^{+}$calcd for $\mathrm{C}_{15} \mathrm{H}_{25} \mathrm{~N}_{2} \mathrm{O}_{2} \mathrm{Pd}^{+}, 371.0951$; found, 371.0947. [M $\left.+\mathrm{H}\right]^{+}$calcd for $\mathrm{C}_{15} \mathrm{H}_{26} \mathrm{IN}_{2} \mathrm{O}_{2} \mathrm{Pd}^{+}$, 499.0068; found, 499.0075. [M $\left.+\mathrm{Na}\right]^{+}$calcd for $\mathrm{C}_{15} \mathrm{H}_{25} \mathrm{IN}_{2} \mathrm{NaO}_{2} \mathrm{Pd}^{+}$, 520.9888; found, 520.9892. [M $+\mathrm{K}]^{+}$calcd for $\mathrm{C}_{15} \mathrm{H}_{25} \mathrm{IKN}_{2} \mathrm{O}_{2} \mathrm{Pd}^{+}, 536.9627$; found, 536.9628 .

Elemental analysis (\%): calcd for $\mathrm{C}_{15} \mathrm{H}_{25} \mathrm{IN}_{2} \mathrm{O}_{2} \mathrm{Pd}, \mathrm{C} 36.1, \mathrm{H} \mathrm{5.05}, \mathrm{N} 5.62$; found, C 36.1, H 5.07, N 5.64.

M.p.: $180{ }^{\circ} \mathrm{C}$ (decomp.)

cis-(tmeda) $\operatorname{Pd}\left(p-F_{3} C C_{6} H_{4}\right) I^{2}\left(\right.$ pre- $\left.C_{3}\right)$<smiles>CN1CCN(C)[Pb]1(I)c1ccc(C(F)(F)F)cc1</smiles>

According to the general procedure, the reaction of $\mathrm{Pd}_{2} \mathrm{dba}_{3} \cdot \mathrm{CHCl}_{3}(3.01 \mathrm{~g}, 2.90 \mathrm{mmol})$, TMEDA (1.13 mL, $7.54 \mathrm{mmol})$ and 4-iodobenzotrifluoride $(1.24 \mathrm{~mL}, 8.41 \mathrm{mmol})$ in toluene $(40.0 \mathrm{~mL})$ provided the complex $(2.34 \mathrm{~g}, 4.73 \mathrm{mmol}, 82 \%)$ as yellow solid.

${ }^{1} \mathrm{H}$ NMR $\left(400 \mathrm{MHz}, \mathrm{CDCl}_{3}\right): \delta 7.42(\mathrm{~d}, J=8.1 \mathrm{~Hz}, 2 \mathrm{H}), 7.15(\mathrm{~d}, J=7.8 \mathrm{~Hz}, 2 \mathrm{H}), 2.76-2.72(\mathrm{~m}, 2 \mathrm{H}), 2.70(\mathrm{~s}, 6 \mathrm{H})$, 2.60-2.56 (m, $2 \mathrm{H}), 2.33(\mathrm{~s}, 6 \mathrm{H}) \mathrm{ppm}$.

${ }^{13} \mathrm{CNMR}\left(101 \mathrm{MHz} \mathrm{CDCl}_{3}\right): \delta 152.2,136.7,125.3(\mathrm{q}, J=32 \mathrm{~Hz}), 125.1(\mathrm{q}, J=271 \mathrm{~Hz}), 122.5(\mathrm{q}, J=3.8 \mathrm{~Hz}), 62.4$, $58.5,50.1,50.0 \mathrm{ppm}$.

${ }^{19} \mathrm{~F}$ NMR $\left(376 \mathrm{MHz} \mathrm{CDCl}_{3}\right): \delta-61.6 \mathrm{ppm}$.

IR $\left(\nu / \mathrm{cm}^{-1}\right)$ : $3003(\mathrm{w}), 2978(\mathrm{w}), 2887(\mathrm{w}), 2842(\mathrm{w}), 1584(\mathrm{~m}), 1455(\mathrm{~m}), 1381(\mathrm{w}), 1318(\mathrm{~m}), 1282(\mathrm{~m}), 1178(\mathrm{w})$, $1153(\mathrm{~m}), 1125(\mathrm{~s}), 1094(\mathrm{~s}), 1069(\mathrm{~s}), 1006(\mathrm{~m}), 955(\mathrm{~m}), 931(\mathrm{w}), 841(\mathrm{w}), 823(\mathrm{~m}), 800(\mathrm{~s}), 768(\mathrm{~m}), 730(\mathrm{~m}), 681$ 
$(\mathrm{m})$.

HRESI-MS $(\mathrm{MeOH},+, \mathrm{m} / \mathrm{z}):[\mathrm{M}+\mathrm{Na}]^{+}$calcd for $\mathrm{C}_{13} \mathrm{H}_{20} \mathrm{~F}_{3} \mathrm{IN}_{2} \mathrm{NaPd}^{+}$, 516.9550; found, 516.9552.

Elemental analysis (\%): calcd for $\mathrm{C}_{13} \mathrm{H}_{20} \mathrm{~F}_{3} \mathrm{IN}_{2} \mathrm{Pd}, \mathrm{C} 31.7, \mathrm{H}$ 4.16, N 5.68; found, C 31.6, H 4.08, N 5.66.

M.p.: $176{ }^{\circ} \mathrm{C}$ (decomp.)

\section{cis-(tmeda) $\left.\operatorname{Pd}\left(p-\mathrm{NCC}_{6} \mathrm{H}_{4}\right)\right|^{2}$ (pre-CN)}<smiles>C[N+]1(C)CCN(I)[P@]1(I)c1ccc(C#N)cc1</smiles>

According to the general procedure, the reaction of $\mathrm{Pd}_{2} \mathrm{dba}_{3} \cdot \mathrm{CHCl}_{3}(1.56 \mathrm{~g}, 1.51 \mathrm{mmol})$, TMEDA $(0.56 \mathrm{~mL}, 3.74 \mathrm{mmol})$ and 4-iodobenzonitrile $(0.98 \mathrm{~g}, 4.30 \mathrm{mmol})$ in toluene $(30.0 \mathrm{~mL})$ provided the complex $(1.17 \mathrm{~g}, 2.59 \mathrm{mmol}, 86 \%)$ as tan solid.

${ }^{1} \mathrm{H}$ NMR $\left(400 \mathrm{MHz}, \mathrm{CDCl}_{3}\right): \delta 7.45(\mathrm{~d}, J=8.3 \mathrm{~Hz}, 2 \mathrm{H}), 7.15(\mathrm{~d}, J=8.3 \mathrm{~Hz}, 2 \mathrm{H}), 2.76-2.71(\mathrm{~m}, 2 \mathrm{H}), 2.68(\mathrm{~s}, 6 \mathrm{H})$, 2.60-2.55 (m, $2 \mathrm{H}), 2.32(\mathrm{~s}, 6 \mathrm{H}) \mathrm{ppm}$.

${ }^{13} \mathrm{C}$ NMR $\left(101 \mathrm{MHz}, \mathrm{CDCl}_{3}\right): \delta 157.1,137.6,128.7,120.2,106.1,62.3,58.5,50.2,50.1 \mathrm{ppm}$.

IR $\left(\nu / \mathrm{cm}^{-1}\right)$ : $3001(\mathrm{w}), 2973(\mathrm{w}), 2886(\mathrm{w}), 2841(\mathrm{w}), 2218(\mathrm{~m}), 1569(\mathrm{~m}), 1470(\mathrm{~m}), 1453(\mathrm{~m}), 1377(\mathrm{w}), 1281(\mathrm{w})$, $1171(w), 1123(w), 1093(w), 1064(w), 1054(m), 1041(w), 1011(m), 955(m), 929(w), 818(s), 802(s), 268(m)$, $713(\mathrm{w})$.

HRESI-MS $(\mathrm{MeOH},+, \mathrm{m} / \mathrm{z})$ : $[\mathrm{M}+\mathrm{Na}]^{+}$calcd for $\mathrm{C}_{13} \mathrm{H}_{20} \mathrm{IN}_{3} \mathrm{NaPd}^{+}, 473.9629$; found, 473.9633 .

Elemental analysis (\%): calcd for $\mathrm{C}_{13} \mathrm{H}_{20} \mathrm{IN}_{3} \mathrm{Pd}, \mathrm{C} 34.6, \mathrm{H} 4.46, \mathrm{~N}$ 9.30; found, C 34.9, H 4.73, N 9.34.

M.p.: $169{ }^{\circ} \mathrm{C}$ (decomp.)

\section{3 cis-(dppe) $\operatorname{Pd}(\mathrm{Ar}) \mid$ complexes}

General procedure: A solution of the tmeda-ligated arylpalladium(II) complex (1 equiv.) and dppe (1 equiv.) in DCM was stirred at room temperature for approx. $10 \mathrm{~min}$. Then $\mathrm{Et}_{2} \mathrm{O}(70.0 \mathrm{~mL})$ was added, which resulted in the precipitation of a solid. The supernatant liquid was decanted, the solid washed with $\mathrm{Et}_{2} \mathrm{O}$ and dried in vacuum.

\section{cis-(dppe) $\mathrm{Pd}\left(p-\mathrm{MeOC}_{6} \mathrm{H}_{4}\right) \mathrm{I}^{6}(\mathrm{OMe})$}<smiles>COc1ccc([Pb]2(I)CCCP2c2ccccc2)cc1</smiles>

According to the general procedure, the reaction of complex pre-OMe $(456 \mathrm{mg}$, $1.00 \mathrm{mmol})$, dppe $(401 \mathrm{mg}, 1.01 \mathrm{mmol})$ in $\mathrm{DCM}(5.00 \mathrm{~mL})$ provided the complex $(735 \mathrm{mg}$, $1.00 \mathrm{mmol}$, quant.) as pale yellow solid.

${ }^{1} \mathrm{H} \mathrm{NMR}\left(400 \mathrm{MHz}, \mathrm{CDCl}_{3}\right): \delta 7.90(\mathrm{tt}, J=7.6,2.3 \mathrm{~Hz}, 4 \mathrm{H}), 7.48-7.29(\mathrm{~m}, 16 \mathrm{H}), 6.92(\mathrm{td}, J=8.6,2.2 \mathrm{~Hz}, 2 \mathrm{H})$, $6.42(\mathrm{dd}, J=8.5,1.9 \mathrm{~Hz}, 2 \mathrm{H}), 3.61(\mathrm{~s}, 3 \mathrm{H}), 2.36(\mathrm{dt}, J=27.1,7.5 \mathrm{~Hz}, 2 \mathrm{H}), 2.29-2.14(\mathrm{~m}, 2 \mathrm{H}) \mathrm{ppm}$.

${ }^{31} \mathrm{P}$ NMR $\left(162 \mathrm{MHz}, \mathrm{CDCl}_{3}\right): \delta 49.91(\mathrm{~d}, J=26 \mathrm{~Hz}), 35.21(\mathrm{~d}, J=26 \mathrm{~Hz}) \mathrm{ppm}$. IR $\left(\nu / \mathrm{cm}^{-1}\right)$ : $3059(\mathrm{vw}), 3004(\mathrm{vw}), 2923(\mathrm{vw}), 2828(\mathrm{vw}), 1573(\mathrm{w}), 1477(\mathrm{~m}), 1433(\mathrm{~m}), 1413(\mathrm{w}), 1306(\mathrm{w}), 1259(\mathrm{~m})$, $1231(\mathrm{~s}), 1173(\mathrm{~m}), 1102(\mathrm{~m}), 1023(\mathrm{~m}), 1002(\mathrm{~m}), 931(\mathrm{w}), 876(\mathrm{~m}), 849(\mathrm{w}), 820(\mathrm{~m}), 803(\mathrm{~m}), 782(\mathrm{w}), 749(\mathrm{~s}), 739$ $(\mathrm{m}), 704(\mathrm{~s}), 689(\mathrm{~s}), 675(\mathrm{~s})$.

HRESI-MS (DCM/MeOH,,$+ \mathrm{m} / z)$ : [M - I $]^{+}$calcd for $\mathrm{C}_{33} \mathrm{H}_{31} \mathrm{OP}_{2} \mathrm{Pd}^{+}$, 611.0885; found, 611.0868. [M - $\left.\mathrm{Ar}\right]^{+}$630.9407; calcd for $\mathrm{C}_{26} \mathrm{H}_{24} \mathrm{IP}_{2} \mathrm{Pd}^{+}$, 630.9433. [M + Na] $]^{+} 760.9791$; calcd for $\mathrm{C}_{33} \mathrm{H}_{31} 1 \mathrm{NaOP}_{2} \mathrm{Pd}^{+}, 760.9822$. 
Elemental analysis (\%): calcd for $\mathrm{C}_{33} \mathrm{H}_{31} \mathrm{IOP}_{2} \mathrm{Pd}, \mathrm{C}$ 53.6, H 4.23; found, C 53.7, H 4.36.

M.p.: $144{ }^{\circ} \mathrm{C}$ (decomp.)

\section{cis-(dppe) $\mathrm{Pd}\left(p-\mathrm{MeC}_{6} \mathrm{H}_{4}\right) I^{6}(\mathrm{Me})$}<smiles></smiles>

According to the general procedure, the reaction of complex pre-Me $(446 \mathrm{mg}, 1.01 \mathrm{mmol})$, dppe $(420 \mathrm{mg}, 1.05 \mathrm{mmol})$ in DCM $(5.00 \mathrm{~mL})$ provided the complex $(736 \mathrm{mg}, 1.02 \mathrm{mmol}$, quant.) as pale yellow solid.

${ }^{1} \mathrm{H} \operatorname{NMR}\left(400 \mathrm{MHz}, \mathrm{CDCl}_{3}\right): \delta 7.95-7.84(\mathrm{~m}, 4 \mathrm{H}), 7.49-7.41(\mathrm{~m}, 8 \mathrm{H}), 7.41-7.27(\mathrm{~m}, 8 \mathrm{H}), 6.93(\mathrm{t}, J=7.1 \mathrm{~Hz}, 2 \mathrm{H})$, $6.58(\mathrm{~d}, J=7.3 \mathrm{~Hz}, 2 \mathrm{H}), 2.43-2.28(\mathrm{~m}, 2 \mathrm{H}), 2.29-2.13(\mathrm{~m}, 2 \mathrm{H}), 2.09(\mathrm{~s}, 3 \mathrm{H}) \mathrm{ppm}$.

${ }^{31} \mathrm{P}$ NMR $\left(162 \mathrm{MHz}, \mathrm{CDCl}_{3}\right): \delta 49.32(\mathrm{~d}, J=28 \mathrm{~Hz}), 34.44(\mathrm{~d}, J=28 \mathrm{~Hz}) \mathrm{ppm}$.

IR $\left(\nu / \mathrm{cm}^{-1}\right)$ : $3048(w), 3006(w), 2938(w), 2912(w), 1572(w), 1478(\mathrm{~m}), 1434(\mathrm{~s}), 1406(w), 1307(w), 1234(w)$, $1206(\mathrm{w}), 1183(\mathrm{w}), 1160(\mathrm{w}), 1099(\mathrm{~s}), 1051(\mathrm{w}), 1027(\mathrm{w}), 1010(\mathrm{~m}), 998(\mathrm{~m}), 879(\mathrm{~m}), 825(\mathrm{~m}), 791(\mathrm{~s}), 740(\mathrm{~s}), 703$ (s), $688(\mathrm{~s}), 657(\mathrm{~s})$.

HRESI-MS (DCM/MeOH,,$+ \mathrm{m} / \mathrm{z})$ : $[\mathrm{M}-\mathrm{I}]^{+}$calcd for $\mathrm{C}_{33} \mathrm{H}_{31} \mathrm{P}_{2} \mathrm{Pd}^{+}$, 595.0936; found, 595.0910. [M $\left.+\mathrm{Na}\right]^{+}$calcd for $\mathrm{C}_{33} \mathrm{H}_{31} \mathrm{INaP}_{2} \mathrm{Pd}^{+}, 744.9873$; found, 744.9835. [M $\left.+\mathrm{K}\right]^{+}$calcd for $\mathrm{C}_{33} \mathrm{H}_{31} 1 \mathrm{KP}_{2} \mathrm{Pd}^{+}, 760.9612$; found, 760.9574 .

Elemental analysis (\%): calcd for $\mathrm{C}_{33} \mathrm{H}_{31} \mathrm{IP}_{2} \mathrm{Pd}, \mathrm{C} 54.8, \mathrm{H}$ 4.32; found, C 54.9, H 4.64.

M.p.: $143{ }^{\circ} \mathrm{C}$ (decomp.)<smiles>CC(C)(C)C(P)C(C)(C)C</smiles>

According to the general procedure, the reaction of complex pre-H $(283 \mathrm{mg}, 0.66 \mathrm{mmol})$, dppe $(270 \mathrm{mg}, 0.68 \mathrm{mmol})$ in DCM $(5.00 \mathrm{~mL})$ provided the complex $(469 \mathrm{mg}, 0.66 \mathrm{mmol}$, quant.) as pale yellow solid.

${ }^{1} \mathrm{H} \mathrm{NMR}\left(400 \mathrm{MHz}, \mathrm{CDCl}_{3}\right): \delta 7.90(\mathrm{ddd}, J=9.9,5.6,2.2 \mathrm{~Hz}, 4 \mathrm{H}), 7.50-7.41(\mathrm{~m}, 8 \mathrm{H}), 7.40-7.28(\mathrm{~m}, 8 \mathrm{H}), 7.07$ (td, $J=6.9,3.8 \mathrm{~Hz}, 2 \mathrm{H}), 6.73(\mathrm{td}, J=7.4,2.6 \mathrm{~Hz}, 2 \mathrm{H}), 6.64(\mathrm{t}, J=6.7 \mathrm{~Hz}, 1 \mathrm{H}), 2.46-2.28(\mathrm{~m}, 2 \mathrm{H}), 2.29-2.12(\mathrm{~m}, 2$ H) $\mathrm{ppm}$.

${ }^{31} \mathrm{P}$ NMR $\left(162 \mathrm{MHz}, \mathrm{CDCl}_{3}\right): \delta 49.55(\mathrm{~d}, J=28 \mathrm{~Hz}), 34.54(\mathrm{~d}, J=28 \mathrm{~Hz}) \mathrm{ppm}$.

IR $\left(\nu / \mathrm{cm}^{-1}\right)$ : $3048(\mathrm{w}), 2968(\mathrm{vw}), 2909(\mathrm{vw}), 1562(\mathrm{w}), 1481(\mathrm{w}), 1468(\mathrm{w}), 1434(\mathrm{~m}), 1404(\mathrm{w}), 1308(\mathrm{w}), 1185(\mathrm{w})$, $1157(\mathrm{w}), 1102(\mathrm{~m}), 1056(\mathrm{w}), 1015(\mathrm{w}), 995(\mathrm{w}), 874(\mathrm{~m}), 819(\mathrm{~m}), 744(\mathrm{~m}), 726(\mathrm{~m}), 689(\mathrm{~s}), 675(\mathrm{~s})$.

HRESI-MS (DCM/MeOH,,$+ \mathrm{m} / \mathrm{z}):[\mathrm{M}-\mathrm{I}]^{+}$calcd for $\mathrm{C}_{32} \mathrm{H}_{29} \mathrm{P}_{2} \mathrm{Pd}^{+}, 581.0779$; found, 581.0738. [M $\left.+\mathrm{Na}\right]^{+}$calcd for $\mathrm{C}_{32} \mathrm{H}_{29} \mathrm{INaP}_{2} \mathrm{Pd}^{+}, 730.9716$; found, 730.9665 .

Elemental analysis (\%): calcd for $\mathrm{C}_{32} \mathrm{H}_{29} \mathrm{IP}_{2} \mathrm{Pd}, \mathrm{C} 54.2, \mathrm{H}$ 4.12; found, C 54.7, H 4.46.

M.p.: $179-181^{\circ} \mathrm{C}$ (decomp.) 


\section{cis-(dppe) $\mathrm{Pd}\left(p-\mathrm{FC}_{6} \mathrm{H}_{4}\right) I(\mathrm{~F})$}<smiles></smiles>

According to the general procedure, the reaction of complex pre- $\mathbf{F}(445 \mathrm{mg}, 1.00 \mathrm{mmol})$, dppe $(399 \mathrm{mg}, 1.00 \mathrm{mmol})$ in DCM $(5.00 \mathrm{~mL})$ provided the complex $(640 \mathrm{mg}, 0.88 \mathrm{mmol}$, $88 \%)$ as pale yellow solid. Crystallization from a solution in DCM by slow diffusion with pentane provided single crystals suitable for $\mathrm{X}$-ray crystallographic analysis.

${ }^{1} \mathrm{H}$ NMR $\left(400 \mathrm{MHz}, \mathrm{CD}_{2} \mathrm{Cl}_{2}\right): \delta 7.89(\mathrm{ddt}, J=10.7,7.6,1.5 \mathrm{~Hz}, 4 \mathrm{H}), 7.56-7.45(\mathrm{~m}, 8 \mathrm{H}), 7.45-7.33(\mathrm{~m}, 8 \mathrm{H}), 6.97$ (qt, $J=8.3,1.8 \mathrm{~Hz}, 2 \mathrm{H}), 6.52(\mathrm{ddd}, J=10.4,8.6,2.0 \mathrm{~Hz}, 2 \mathrm{H}), 2.43(\mathrm{dtd}, J=27.6,8.4,8.0,5.2 \mathrm{~Hz}, 2 \mathrm{H}), 2.25$ (dtd, $J=28.0,8.8,8.1,5.5 \mathrm{~Hz}, 2 \mathrm{H}) \mathrm{ppm}$.

${ }^{31} \mathrm{P}$ NMR $\left(162 \mathrm{MHz}, \mathrm{CD}_{2} \mathrm{Cl}_{2}\right): \delta 51.46(\mathrm{~d}, J=26 \mathrm{~Hz}), 37.03(\mathrm{~d}, J=26 \mathrm{~Hz}) \mathrm{ppm}$.

${ }^{19} \mathrm{~F} \mathrm{NMR}\left(376 \mathrm{MHz}, \mathrm{CD}_{2} \mathrm{Cl}_{2}\right): \delta-124.28 \mathrm{ppm}$.

IR $\left(\nu / \mathrm{cm}^{-1}\right)$ : $3046(\mathrm{w}), 3017(\mathrm{w}), 2954(\mathrm{w}), 2915(\mathrm{w}), 1569(\mathrm{w}), 1476(\mathrm{~m}), 1435(\mathrm{~m}), 1402(\mathrm{w}), 1310(\mathrm{w}), 1214(\mathrm{~m})$, $1155(\mathrm{~m}), 1106(\mathrm{~m}), 1079(\mathrm{w}), 1058(\mathrm{w}), 1044(\mathrm{w}), 1027(\mathrm{w}), 1014(\mathrm{w}), 998(\mathrm{w}), 874(\mathrm{~m}), 818(\mathrm{~m}), 801(\mathrm{~m}), 743(\mathrm{~s})$, $706(\mathrm{~s}), 687(\mathrm{~s})$.

HRESI-MS (DCM/MeOH,,$+ \mathrm{m} / \mathrm{z})$ : [M - I] ${ }^{+}$calcd for $\mathrm{C}_{32} \mathrm{H}_{28} \mathrm{FP}_{2} \mathrm{Pd}^{+}$, 599.0685; found, 599.0659. [M $\left.+\mathrm{Na}\right]^{+}$calcd for $\mathrm{C}_{32} \mathrm{H}_{28} \mathrm{FINaP}_{2} \mathrm{Pd}^{+}, 748.9622$; found, 748.9586 .

Elemental analysis (\%): calcd for $\mathrm{C}_{32} \mathrm{H}_{28} \mathrm{FIP}_{2} \mathrm{Pd}, \mathrm{C} 52.9, \mathrm{H}$ 3.88; found, C 52.9, H 4.02 .

M.p.: $168{ }^{\circ} \mathrm{C}$ (decomp.)

\section{cis-(dppe) $\mathrm{Pd}\left(p-\mathrm{EtO}_{2} \mathrm{CC}_{6} \mathrm{H}_{4}\right) !\left(\mathrm{CO}_{2} \mathrm{Et}\right)$}<smiles>CCOc1ccc([Pb]2(I)CC[Pb]2c2ccccc2)cc1</smiles>

According to the general procedure, the reaction of complex pre- $\mathrm{CO}_{2} \mathrm{Et}(500 \mathrm{mg}$, $1.00 \mathrm{mmol})$, dppe $(400 \mathrm{mg}, 1.00 \mathrm{mmol})$ in DCM $(5.00 \mathrm{~mL})$ provided the complex $(769 \mathrm{mg}$, $0.98 \mathrm{mmol}, 98 \%)$ as pale yellow solid.

${ }^{1} \mathrm{H}$ NMR $\left(400 \mathrm{MHz}, \mathrm{CD}_{2} \mathrm{Cl}_{2}\right): \delta 7.94-7.85(\mathrm{~m}, 4 \mathrm{H}), 7.57-7.45(\mathrm{~m}, 8 \mathrm{H}), 7.45-7.32(\mathrm{~m}, 10 \mathrm{H}), 7.21(\mathrm{tt}, J=8.1,1.9$ $\mathrm{Hz}, 2 \mathrm{H}), 4.24(\mathrm{q}, J=7.1 \mathrm{~Hz}, 2 \mathrm{H}), 2.45(\mathrm{dtd}, J=28.2,8.8,5.4 \mathrm{~Hz}, 2 \mathrm{H}), 2.27(\mathrm{dtd}, J=28.2,8.7,8.2,5.5 \mathrm{~Hz}, 2 \mathrm{H})$, $1.30(\mathrm{t}, J=7.1 \mathrm{~Hz}, 3 \mathrm{H}) \mathrm{ppm}$.

${ }^{31} \mathrm{P}$ NMR $\left(162 \mathrm{MHz}, \mathrm{CD}_{2} \mathrm{Cl}_{2}\right): \delta 50.96(\mathrm{dd}, J=26 \mathrm{~Hz}), 36.96(\mathrm{~d}, J=26 \mathrm{~Hz}) \mathrm{ppm}$.

IR $\left(\nu / \mathrm{cm}^{-1}\right)$ : $3053(\mathrm{w}), 2984(\mathrm{w}), 2943(\mathrm{w}), 1699(\mathrm{~s}), 1577(\mathrm{~m}), 1482(\mathrm{w}), 1433(\mathrm{~m}), 1388(\mathrm{w}), 1366(\mathrm{w}), 1305(\mathrm{w})$, $1282(\mathrm{~s}), 1173(\mathrm{~m}), 1112(\mathrm{~s}), 1100(\mathrm{~s}), 105(\mathrm{w}), 1013(\mathrm{~m}), 998(\mathrm{w}), 869(\mathrm{~m}), 838(\mathrm{~m}), 819(\mathrm{~m}), 783(\mathrm{w}), 747(\mathrm{~s}), 739$ (s), $690(\mathrm{~s}), 674(\mathrm{~s})$.

HRESI-MS (DCM/MeOH,,$+ \mathrm{m} / \mathrm{z})$ : [(M - I $]^{+}$calcd for $\mathrm{C}_{35} \mathrm{H}_{33} \mathrm{O}_{2} \mathrm{P}_{2} \mathrm{Pd}^{+}$, 653.0991; found, 653.1025. [M $\left.+\mathrm{Na}\right]^{+}$calcd for $\mathrm{C}_{35} \mathrm{H}_{33} \mathrm{INaO}_{2} \mathrm{P}_{2} \mathrm{Pd}^{+}$, 802.9928; found, 802.9970. [2M - I] calcd for $\mathrm{C}_{70} \mathrm{H}_{66} \mathrm{IO}_{4} \mathrm{P}_{4} \mathrm{Pd}_{2}{ }^{+}, 1435.1030$; found, 1435.1088 . Elemental analysis (\%): calcd for $\mathrm{C}_{35} \mathrm{H}_{33} \mathrm{IO}_{2} \mathrm{P}_{2} \mathrm{Pd}, \mathrm{C} 53.8, \mathrm{H}$ 4.26; found, $\mathrm{C} 53.9, \mathrm{H} 4.54$.

M.p.: $166-168{ }^{\circ} \mathrm{C}$ (decomp.) 


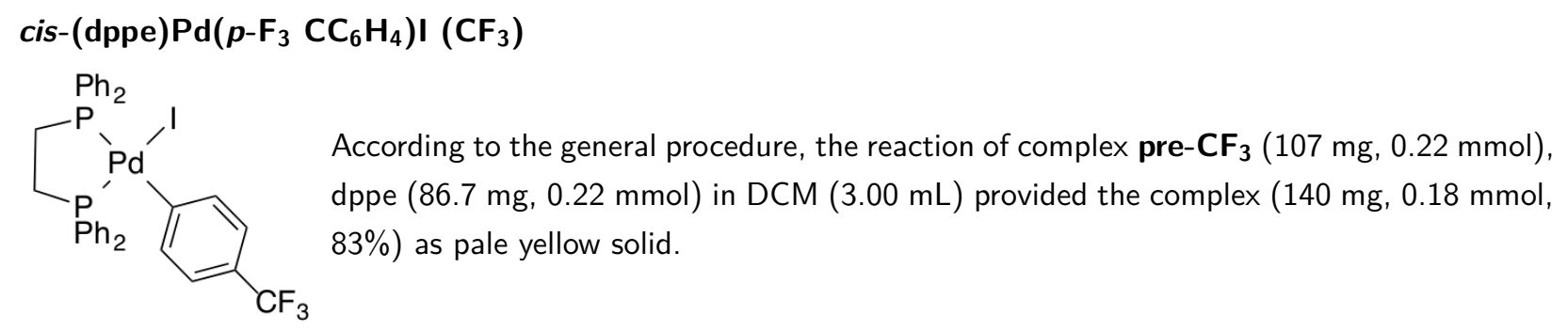

${ }^{1} \mathrm{H}$ NMR $\left(400 \mathrm{MHz}, \mathrm{CDCl}_{3}\right): \delta 7.94-7.85(\mathrm{~m}, 4 \mathrm{H}), 7.53-7.43(\mathrm{~m}, 8 \mathrm{H}), 7.39-7.29(\mathrm{~m}, 8 \mathrm{H}), 7.21(\mathrm{t}, J=7.5 \mathrm{~Hz}, 2 \mathrm{H})$, $6.93(\mathrm{~d}, J=7.7 \mathrm{~Hz}, 2 \mathrm{H}), 2.40(\mathrm{dq}, J=26.1,7.7,7.0 \mathrm{~Hz}, 2 \mathrm{H}), 2.23(\mathrm{dtd}, J=28.0,8.8,8.0,5.3 \mathrm{~Hz}, 2 \mathrm{H}) \mathrm{ppm}$.

${ }^{31} \mathrm{P}$ NMR $\left(162 \mathrm{MHz}, \mathrm{CDCl}_{3}\right): \delta 50.97(\mathrm{~d}, J=27 \mathrm{~Hz}), 36.24(\mathrm{~d}, J=27 \mathrm{~Hz}) \mathrm{ppm}$.

${ }^{19} \mathrm{~F} \mathrm{NMR}\left(376 \mathrm{MHz}, \mathrm{CDCl}_{3}\right): \delta-61.69 \mathrm{ppm}$.

IR $\left(\nu / \mathrm{cm}^{-1}\right)$ : $3063(\mathrm{w}), 3043(\mathrm{w}), 3013(\mathrm{w}), 2949(\mathrm{w}), 2913(\mathrm{w}), 1586(\mathrm{~m}), 1482(\mathrm{w}), 1433(\mathrm{~m}), 1412(\mathrm{w}), 1385(\mathrm{w})$, 1325 (s), 1269 (w), $1159(\mathrm{~m}), 1099$ (s), 1091 (s), 1065 (s), 1041 (w), 1027 (w), 1007 (m), 875 (m), 848 (w), 823 (s), $813(\mathrm{~s}), 750(\mathrm{~s}), 742(\mathrm{~m}), 725(\mathrm{w}), 703(\mathrm{~s}), 690(\mathrm{~s}), 677(\mathrm{~s})$.

HRESI-MS (DCM/MeOH,,$+ \mathrm{m} / \mathrm{z}$ ): [M - I $]^{+}$calcd for $\mathrm{C}_{33} \mathrm{H}_{28} \mathrm{~F}_{3} \mathrm{P}_{2} \mathrm{Pd}^{+}, 649.0653$; found, 649.0680. [M $\left.+\mathrm{Na}\right]^{+}$calcd for $\mathrm{C}_{33} \mathrm{H}_{28} \mathrm{~F}_{3} \mathrm{INaP}{ }_{2} \mathrm{Pd}^{+}$, 798.9590; found, 798.9618. [2M - I] calcd for $\mathrm{C}_{66} \mathrm{H}_{56} \mathrm{~F}_{6} \mathrm{IP}_{4} \mathrm{Pd}_{2}{ }^{+}, 1427.0355$; found, 1427.0393. [2 $\mathrm{M}+\mathrm{Na}]^{+}$calcd for $\mathrm{C}_{66} \mathrm{H}_{56} \mathrm{~F}_{6} \mathrm{I}_{2} \mathrm{NaP}_{4} \mathrm{Pd}_{2}{ }^{+}$, 1576.9292; found, 1576.9309 .

Elemental analysis (\%): calcd for $\mathrm{C}_{33} \mathrm{H}_{28} \mathrm{~F}_{3} \mathrm{IP}_{2} \mathrm{Pd}, \mathrm{C}$ 51.0, $\mathrm{H}$ 3.63; found, C 51.1, H 3.97.

M.p.: $163-164{ }^{\circ} \mathrm{C}$ (decomp.)

\section{cis-(dppe) $\mathrm{Pd}\left(p-\mathrm{NCC}_{6} \mathrm{H}_{4}\right) \mathrm{I}(\mathrm{CN})$}<smiles>N#Cc1ccc([PH]2(I)c3ccccc3P3CCP32)cc1</smiles>

According to the general procedure, the reaction of complex pre-CN $(453 \mathrm{mg}, 1.00 \mathrm{mmol})$, dppe $(400 \mathrm{mg}, 1.00 \mathrm{mmol})$ in DCM $(5.00 \mathrm{~mL})$ provided the complex $(665 \mathrm{mg}, 0.91 \mathrm{mmol}$, $91 \%)$ as pale yellow solid.

${ }^{1} \mathrm{H}$ NMR $\left(400 \mathrm{MHz}, \mathrm{CD}_{2} \mathrm{Cl}_{2}\right): \delta 7.88(\mathrm{ddd}, J=10.2,7.5,1.8 \mathrm{~Hz}, 4 \mathrm{H}), 7.59-7.47(\mathrm{~m}, 8 \mathrm{H}), 7.46-7.35(\mathrm{~m}, 8 \mathrm{H}), 7.25$ $(\mathrm{td}, J=7.9,1.8 \mathrm{~Hz}, 2 \mathrm{H}), 6.94(\mathrm{dd}, J=8.0,2.2 \mathrm{~Hz}, 2 \mathrm{H}), 2.46(\mathrm{dtd}, J=27.8,8.5,8.0,5.2 \mathrm{~Hz}, 2 \mathrm{H}), 2.35-2.19(\mathrm{~m}, 2$ H) $\mathrm{ppm}$.

${ }^{31} \mathrm{P}$ NMR $\left(162 \mathrm{MHz}, \mathrm{CD}_{2} \mathrm{Cl}_{2}\right): \delta 52.00(\mathrm{~d}, J=26 \mathrm{~Hz}), 38.11(\mathrm{~d}, J=26 \mathrm{~Hz}) \mathrm{ppm}$.

IR $\left(\nu / \mathrm{cm}^{-1}\right)$ : $3049(\mathrm{w}), 2954(\mathrm{w}), 2913(\mathrm{w}), 2221(\mathrm{~m}), 1574(\mathrm{~m}), 1483(\mathrm{w}), 1473(\mathrm{w}), 1434(\mathrm{~m}), 1404(\mathrm{w}), 1310(\mathrm{w})$, $1233(w), 1181(w), 1159(w), 1104(m), 1059(w), 1047(w), 1026(w), 1013(w), 998(w), 873(m), 820(m), 808(s)$, $743(\mathrm{~s}), 705(\mathrm{~s}), 687(\mathrm{~s}), 675(\mathrm{~s})$.

HRESI-MS (DCM/MeOH,,$+ \mathrm{m} / \mathrm{z}$ ): $[\mathrm{M}-\mathrm{I}]^{+}$calcd for $\mathrm{C}_{33} \mathrm{H}_{28} \mathrm{NP}_{2} \mathrm{Pd}^{+}, 606.0732$; found, 606.0750. $[\mathrm{M}+\mathrm{Na}]^{+}$calcd for $\mathrm{C}_{33} \mathrm{H}_{28} \mathrm{INNaP}{ }_{2} \mathrm{Pd}^{+}$, 755.9669; found, 755.9680. [2M - I] calcd for $\mathrm{C}_{66} \mathrm{H}_{56} \mathrm{IN}_{2} \mathrm{P}_{4} \mathrm{Pd}_{2}{ }^{+}, 1341.0512$; found, 1341.0502. [2M $+\mathrm{Na}]^{+}$calcd for $\mathrm{C}_{66} \mathrm{H}_{56} \mathrm{I}_{2} \mathrm{NaN}_{2} \mathrm{P}_{4} \mathrm{Pd}_{2}{ }^{+}, 1490.9449$; found, 1490.9432 .

Elemental analysis (\%): calcd for $\mathrm{C}_{33} \mathrm{H}_{28} \mathrm{INP}_{2} \mathrm{Pd}, \mathrm{C} 54.0, \mathrm{H} 3.85, \mathrm{~N} 1.91$; found, C 54.3, H 4.38, N 1.93. A matching elemental analysis of this complex was not obtained.

M.p.: $167-168{ }^{\circ} \mathrm{C}$ (decomp.) 


\section{Competition experiments}

General: For the transmetalation study, the arylzinc and arylpalladium complexes need to be in excess to simulate pseudo-zeroth order reaction kinetics. To prevent side reactions, such as homocoupling in the hot GC injector, the excess arylpalladium complexes have to be quenched for instance by reduction using $\mathrm{NH}_{4} \mathrm{HCO}_{2}$ to produce volatile arenes. Samples were measured nine times by GC-FID to identify the relatively large instrumental error as indicated by the standard deviation $\sigma$ in the two tables below. The combination of complexes $\mathbf{H}$ and $\mathbf{F}$ could not be used, because the peaks of the resulting biaryls could not be fully separated in the GC.

Setup: Reaction vials were charged with complexes $\mathbf{X}$ and $\mathbf{Y}$ (each $16 \mu \mathrm{mol}$ ) and a stir bar outside the glovebox. Inside a glovebox, the complexes were dissolved in THF $(10.0 \mathrm{~mL})$ under stirring and a solution of $\mathrm{ZnPh}_{2}(1$ : $22.2 \mathrm{mg}, 0.10 \mathrm{mmol}, 2: 22.2 \mathrm{mg}, 0.10 \mathrm{mmol})$ and $\mathrm{Znl}_{2}(1: 34.0 \mathrm{mg}, 0.11 \mathrm{mmol}, 2: 32.6 \mathrm{mg}, 0.10 \mathrm{mmol})$ in THF $(10 \mathrm{~mL}, \mathrm{c}[\mathrm{PhZnl}]=20.2 \mathrm{mM}, 0.20 \mathrm{~mL}, 4.0 \mu \mathrm{mol})$ was syringed to the complex solutions. The vials were sealed and stirred at room temperature for $1 \mathrm{~h}$ outside the glovebox before a stock solution of TMB (1: $88.0 \mathrm{mg}$, $0.52 \mathrm{mmol}, 2: 84.8 \mathrm{mg}, 0.50 \mathrm{mmol})$ in $\mathrm{MeOH}(50.0 \mathrm{~mL}, \mathrm{c}=10.5 \mathrm{mM}, 0.40 \mathrm{~mL}, 4.0 \mu \mathrm{mol})$ and a spatula tip of $\mathrm{NH}_{4} \mathrm{HCO}_{2}$ were added. The reaction mixtures were stirred at room temperature for $1 \mathrm{~h}$, if necessary, filtrated through a short pad of Celite (pipette), and transferred into GC vials for biaryl quantifications by GC-FID.

Relative reaction constant $k_{\text {rel }}=k_{\mathrm{A}} / k_{\mathrm{B}}$ for reactions

$$
\begin{aligned}
& \mathbf{A}+\mathbf{C} \stackrel{k_{\mathrm{A}}}{\longrightarrow} \mathbf{D} \\
& \mathbf{B}+\mathbf{C} \stackrel{k_{\mathrm{B}}}{\longrightarrow} \mathbf{E}
\end{aligned}
$$

are obtained from the rate laws yielding the relation

$$
k_{\mathrm{rel}}=\frac{\ln \left([\mathbf{A}]_{0}-[\mathbf{D}]\right)-\ln \left([\mathbf{A}]_{0}\right)}{\ln \left([\mathbf{B}]_{0}-[\mathbf{E}]\right)-\ln \left([\mathbf{B}]_{0}\right)}
$$

with initial reactant concentrations $[\mathbf{A}]_{0}$ and $[\mathbf{B}]_{0}$ and product concentrations $[\mathbf{D}]$ and $[\mathbf{E}]$. Initial reactant concentrations are given, while product concentrations are calculated from their ratios and reaction yields. 
Table S1: Results of the competition experiments (first sample) according to the above described procedure. "x/y" refers to the biaryl product ratio obtained from GC-FID.

\begin{tabular}{|c|c|c|c|c|c|c|c|c|c|}
\hline$x$ & $m(m g)$ & $\mathrm{n}(\mu \mathrm{mol})$ & $Y$ & $m(m g)$ & $\mathrm{n}(\mu \mathrm{mol})$ & $x / y$ & $\sigma$ & yield (\%) & $\sigma(\%)$ \\
\hline $\mathrm{OMe}$ & 11.6 & 15.7 & $\mathbf{H}$ & 11.5 & 16.2 & 2.69 & 0.47 & 96 & 8 \\
\hline $\mathrm{Me}$ & 11.9 & 16.5 & $\mathbf{H}$ & 11.2 & 15.8 & 1.78 & 0.17 & 101 & 2 \\
\hline $\mathrm{CO}_{2} \mathrm{Et}$ & 12.6 & 16.1 & $\mathbf{H}$ & 11.5 & 16.2 & 0.13 & 0.02 & 93 & 2 \\
\hline $\mathrm{CF}_{3}$ & 12.7 & 16.3 & $\mathbf{H}$ & 11.3 & 15.9 & 0.18 & 0.00 & 108 & 3 \\
\hline $\mathrm{CN}$ & 11.6 & 15.8 & $\mathbf{H}$ & 11.7 & 16.5 & 0.12 & 0.04 & 84 & 7 \\
\hline Me & 11.7 & 16.2 & $\mathrm{OMe}$ & 11.8 & 16.0 & 0.55 & 0.02 & 84 & 2 \\
\hline $\mathbf{F}$ & 11.3 & 15.5 & $\mathrm{OMe}$ & 11.6 & 15.7 & 0.24 & 0.04 & 92 & 1 \\
\hline $\mathrm{CO}_{2} \mathrm{Et}$ & 12.2 & 15.6 & $\mathrm{OMe}$ & 11.5 & 15.6 & 0.20 & 0.06 & 99 & 5 \\
\hline $\mathrm{CF}_{3}$ & 12.7 & 16.3 & $\mathrm{OMe}$ & 11.5 & 15.6 & 0.00 & 0.00 & 85 & 2 \\
\hline $\mathrm{CN}$ & 11.9 & 16.2 & $\mathrm{OMe}$ & 12.1 & 16.4 & 0.00 & 0.00 & 0 & 0 \\
\hline $\mathbf{F}$ & 11.7 & 16.1 & $\mathrm{Me}$ & 11.8 & 16.3 & 0.43 & 0.03 & 99 & 2 \\
\hline $\mathrm{CO}_{2} \mathrm{Et}$ & 13.5 & 17.3 & Me & 12.4 & 17.2 & 0.04 & 0.03 & 84 & 3 \\
\hline $\mathrm{CF}_{3}$ & 13.3 & 17.1 & Me & 12.9 & 17.8 & 0.00 & 0.00 & 91 & 2 \\
\hline $\mathrm{CN}$ & 13.0 & 17.7 & Me & 12.5 & 17.3 & 0.03 & 0.06 & 84 & 5 \\
\hline $\mathrm{CO}_{2} \mathrm{Et}$ & 12.1 & 15.5 & $\mathbf{F}$ & 11.7 & 16.1 & 0.16 & 0.01 & 101 & 5 \\
\hline $\mathrm{CF}_{3}$ & 12.2 & 15.7 & $F$ & 11.5 & 15.8 & 0.17 & 0.01 & 112 & 10 \\
\hline $\mathrm{CN}$ & 12.0 & 16.4 & $F$ & 11.8 & 16.2 & 0.09 & 0.03 & 98 & 6 \\
\hline $\mathrm{CF}_{3}$ & 12.1 & 15.6 & $\mathrm{CO}_{2} \mathrm{Et}$ & 12.3 & 15.8 & 0.79 & 0.05 & 89 & 3 \\
\hline $\mathrm{CN}$ & 12.0 & 16.4 & $\mathrm{CO}_{2} \mathrm{Et}$ & 12.8 & 16.4 & 0.54 & 0.03 & 83 & 3 \\
\hline $\mathrm{CN}$ & 12.0 & 16.4 & $\mathrm{CF}_{3}$ & 13.0 & 16.7 & 0.75 & 0.04 & 82 & 7 \\
\hline
\end{tabular}


Table S2: Results of the competition experiments (second sample) according to the above described procedure. "x/y" refers to the biaryl product ratio obtained from GC-FID.

\begin{tabular}{|c|c|c|c|c|c|c|c|c|c|}
\hline$x$ & $m(m g)$ & $\mathrm{n}(\mu \mathrm{mol})$ & $Y$ & $m(m g)$ & $\mathrm{n}(\mu \mathrm{mol})$ & $x / y$ & $\sigma$ & yield (\%) & $\sigma(\%)$ \\
\hline $\mathrm{OMe}$ & 12.1 & 16.3 & $\mathbf{H}$ & 11.4 & 16.1 & 3.63 & 0.35 & 83 & 1 \\
\hline $\mathrm{Me}$ & 11.5 & 15.8 & $\mathbf{H}$ & 11.2 & 15.8 & 2.00 & 0.05 & 85 & 1 \\
\hline $\mathrm{CO}_{2} \mathrm{Et}$ & 12.6 & 16.1 & $\mathbf{H}$ & 11.5 & 16.2 & 0.15 & 0.02 & 93 & 1 \\
\hline $\mathrm{CF}_{3}$ & 12.3 & 15.9 & $\mathbf{H}$ & 11.2 & 15.8 & 0.16 & 0.00 & 104 & 2 \\
\hline $\mathrm{CN}$ & 11.8 & 16.1 & $\mathbf{H}$ & 11.4 & 16.0 & 0.09 & 0.15 & 85 & 16 \\
\hline Me & 11.7 & 16.2 & $\mathrm{OMe}$ & 12.0 & 16.2 & 0.50 & 0.01 & 84 & 1 \\
\hline $\mathbf{F}$ & 11.9 & 16.4 & $\mathrm{OMe}$ & 12.0 & 16.3 & 0.26 & 0.01 & 98 & 3 \\
\hline $\mathrm{CO}_{2} \mathrm{Et}$ & 12.5 & 16.0 & $\mathrm{OMe}$ & 11.7 & 15.9 & 0.23 & 0.01 & 104 & 5 \\
\hline $\mathrm{CF}_{3}$ & 12.4 & 16.0 & $\mathrm{OMe}$ & 11.8 & 15.9 & 0.00 & 0.00 & 95 & 3 \\
\hline $\mathrm{CN}$ & 11.6 & 15.9 & $\mathrm{OMe}$ & 11.8 & 16.0 & 0.00 & 0.00 & 80 & 2 \\
\hline $\mathbf{F}$ & 11.5 & 15.9 & $\mathrm{Me}$ & 11.4 & 15.7 & 0.46 & 0.03 & 99 & 1 \\
\hline $\mathrm{CO}_{2} \mathrm{Et}$ & 12.7 & 16.3 & Me & 11.8 & 16.3 & 0.02 & 0.04 & 85 & 3 \\
\hline $\mathrm{CF}_{3}$ & 11.2 & 14.4 & Me & 11.3 & 15.6 & 0.02 & 0.04 & 93 & 2 \\
\hline $\mathrm{CN}$ & 11.6 & 15.7 & Me & 11.4 & 15.7 & 0.00 & 0.00 & 85 & 1 \\
\hline $\mathrm{CO}_{2} \mathrm{Et}$ & 12.8 & 16.3 & $\mathbf{F}$ & 11.9 & 16.4 & 0.18 & 0.01 & 114 & 2 \\
\hline $\mathrm{CF}_{3}$ & 12.6 & 16.3 & $F$ & 11.8 & 16.3 & 0.15 & 0.01 & 108 & 3 \\
\hline $\mathrm{CN}$ & 11.7 & 16.0 & $F$ & 11.6 & 15.9 & 0.00 & 0.00 & 93 & 1 \\
\hline $\mathrm{CF}_{3}$ & 12.8 & 16.4 & $\mathrm{CO}_{2} \mathrm{Et}$ & 12.8 & 16.4 & 0.83 & 0.07 & 97 & 6 \\
\hline $\mathrm{CN}$ & 11.7 & 15.9 & $\mathrm{CO}_{2} \mathrm{Et}$ & 12.5 & 16.0 & 0.56 & 0.06 & 79 & 4 \\
\hline $\mathrm{CN}$ & 14.9 & 15.9 & $\mathrm{CF}_{3}$ & 15.9 & 20.5 & 0.60 & 0.08 & 81 & 3 \\
\hline
\end{tabular}




\section{Additional computational results}

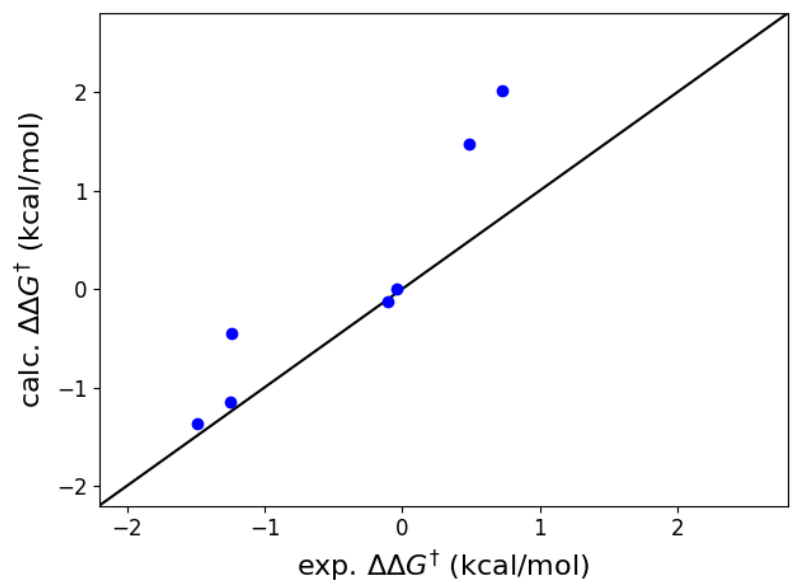

Figure S1: Comparison of experimental and calculated data regarding $\Delta \Delta G^{\ddagger}$ with $\Delta \Delta G^{\ddagger}$ set to zero for $\mathbf{H}$.

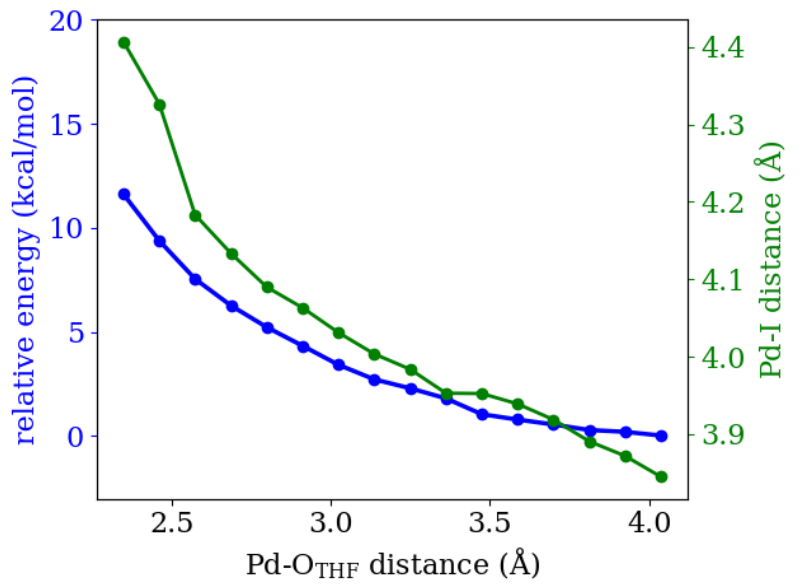

Figure S2: Relaxed potential energy surface for the $\mathrm{Pd}-\mathrm{O}_{\mathrm{THF}}$ distance coupled with simultaneous iodide abstraction. 
Table S3: Selected examples of structural features ( $c f$. Fig. 5 in the main text) changing during the entire transmetalation reaction (in $\AA$ ). $\sigma_{\text {ar }}$ is the standard deviation from the average C-C bond distance in the transferred phenyl ring and used as an indicator for the preservation of aromaticity during the reaction.

\section{OMe}

\begin{tabular}{cccccccc}
\hline & $\mathrm{Pd}-\mathrm{Zn}$ & $\mathrm{Pd}-\mathrm{I}$ & $\mathrm{Zn}-\mathrm{I}$ & $\mathrm{Pd}-\mathrm{C}_{\mathrm{Ph}}$ & $\mathrm{Zn}-\mathrm{C}_{\mathrm{Ph}}$ & $\mathrm{Zn}-\mathrm{O}_{\mathrm{THF}}$ & $\sigma_{\mathrm{ar}}$ \\
\hline I & - & 2.715 & - & - & 1.990 & 2.142 & 0.0113 \\
Ila & 4.466 & 2.720 & 3.624 & 3.803 & 1.994 & 2.261 & 0.0114 \\
IIla & 3.294 & 2.748 & 2.976 & 3.370 & 2.002 & 3.272 & 0.0105 \\
IV & 2.987 & 2.748 & 2.771 & 2.227 & 2.155 & 3.182 & 0.0190 \\
V & 5.669 & 4.981 & 2.598 & 2.056 & 5.839 & 2.034 & 0.008 \\
VI & - & - & 2.586 & 2.049 & - & 2.066 & 0.0075 \\
\hline
\end{tabular}

Me

\begin{tabular}{cccccccc}
\hline & $\mathrm{Pd}-\mathrm{Zn}$ & $\mathrm{Pd}-\mathrm{I}$ & $\mathrm{Zn}-\mathrm{I}$ & $\mathrm{Pd}-\mathrm{C}_{\mathrm{Ph}}$ & $\mathrm{Zn}-\mathrm{C}_{\mathrm{Ph}}$ & $\mathrm{Zn}-\mathrm{O}_{\mathrm{THF}}$ & $\sigma_{\mathrm{ar}}$ \\
\hline I & - & 2.714 & - & - & 1.990 & 2.142 & 0.0113 \\
Ila & 4.471 & 2.719 & 3.635 & 3.807 & 1.994 & 2.259 & 0.011 \\
IIIa & 3.247 & 2.741 & 2.979 & 3.373 & 2.002 & 3.327 & 0.011 \\
IV & 3.086 & 3.718 & 2.825 & 2.249 & 2.136 & 2.812 & 0.017 \\
V & 5.703 & 5.025 & 2.597 & 2.057 & 5.847 & 2.033 & 0.008 \\
VI & - & - & 2.586 & 2.048 & - & 2.066 & 0.008 \\
\hline
\end{tabular}

\section{$\mathbf{F}$}

\begin{tabular}{cccccccc} 
& Pd-Zn & Pd-I & Zn-I & Pd-C & Zn- & Ph & Zn-O \\
\hline I & - & 2.712 & - & - & 1.990 & 2.142 & 0.0113 \\
Ila & 4.476 & 2.717 & 3.641 & 3.806 & 1.994 & 2.254 & 0.0115 \\
IIIa & 3.287 & 2.748 & 2.937 & 3.367 & 2.001 & 3.422 & 0.0106 \\
IV & 3.135 & 3.676 & 2.865 & 2.231 & 2.168 & 2.561 & 0.0193 \\
V & 5.657 & 4.990 & 2.597 & 2.057 & 5.827 & 2.034 & 0.0081 \\
VI & - & - & 2.586 & 2.049 & - & 2.066 & 0.0073 \\
\hline
\end{tabular}

\section{$\mathrm{CO}_{2} \mathrm{Et}$}

\begin{tabular}{cccccccc} 
& Pd-Zn & Pd-I & Zn-I & Pd-C $C_{\text {Ph }}$ & Zn- $C_{\text {Ph }}$ & Zn-O & \\
THF & $\sigma_{\text {ar }}$ \\
\hline I & - & 2.708 & - & - & 1.9898 & 2.142 & 0.0113 \\
Ila & 4.479 & 2.715 & 3.668 & 3.804 & 1.9940 & 2.248 & 0.0115 \\
IIla & 4.331 & 2.738 & 2.988 & 3.425 & 1.995 & 3.216 & 0.0112 \\
IV & 3.108 & 3.554 & 2.867 & 2.269 & 2.1402 & 2.631 & 0.0189 \\
V & 5.752 & 4.982 & 2.599 & 2.0588 & 5.843 & 2.036 & 0.0075 \\
VI & - & - & 2.586 & 2.051 & - & 2.066 & 0.0071 \\
\hline
\end{tabular}

\section{$\mathrm{CF}_{3}$}

\begin{tabular}{cccccccc} 
& Pd-Zn & Pd-I & Zn-I & Pd-C & Zn- & Ph & Zn-O \\
THF & $\sigma_{\text {ar }}$ \\
\hline I & - & 2.707 & - & - & 1.990 & 2.142 & 0.0113 \\
Ila & 4.483 & 2.715 & 3.662 & 3.805 & 1.994 & 2.247 & 0.0115 \\
IIla & 3.256 & 2.742 & 2.977 & 3.380 & 2.002 & 3.311 & 0.0109 \\
IV & 3.186 & 3.682 & 2.876 & 2.218 & 2.181 & 2.495 & 0.0193 \\
V & 5.700 & 4.980 & 2.597 & 2.058 & 5.829 & 2.035 & 0.0078 \\
VI & - & - & 2.586 & 2.051 & - & 2.066 & 0.0071 \\
\hline
\end{tabular}

CN

\begin{tabular}{|c|c|c|c|c|c|c|c|}
\hline & $\mathrm{Pd}-\mathrm{Zn}$ & Pd-I & Zn-I & $\mathrm{Pd}-\mathrm{C}_{\mathrm{Ph}}$ & $\mathrm{Zn}-\mathrm{C}_{\mathrm{Ph}}$ & $\mathrm{Zn}-\mathrm{O}_{\mathrm{THF}}$ & $\sigma_{\mathrm{ar}}$ \\
\hline I & - & 2.707 & - & - & 1.990 & 2.142 & 0.0113 \\
\hline Ila & 4.489 & 2.714 & 3.677 & 3.805 & 1.994 & 2.239 & 0.0117 \\
\hline IIla & 3.310 & 2.741 & 2.979 & 3.373 & 2.000 & 3.327 & 0.0107 \\
\hline IV & 3.198 & 3.560 & 2.894 & 2.238 & 2.177 & 2.434 & 0.0200 \\
\hline $\mathrm{V}$ & 5.695 & 4.957 & 2.597 & 2.058 & 5.840 & 2.036 & 0.0075 \\
\hline VI & - & - & 2.586 & 2.052 & - & 2.066 & 0.0069 \\
\hline
\end{tabular}




\section{Notes on the transition state structure}

To gain more insight into the formation of the TS structure (IIla $\rightarrow$ IV), we investigated at what point the phenyl ring and the iodide ligand are transferred. Fig. S3 shows the relaxed PES for the variation of the $P d-C_{P h}$ and $\mathrm{Zn}$-I distances near the TS structure. Obviously, the transfer of the two ligands is coupled and not strictly successive (Fig. S4). The differences in $\Delta H$ and $\Delta S$ among the various intermediates and the TS are usually below $1 \mathrm{kcal} / \mathrm{mol}$ as long as the separated reactants $(\mathrm{I})$ and products $(\mathrm{VI})$ are disregarded. This suggests that it is sufficient to examine electronic energies instead of calculating more demanding free enthalpies. It becomes immediately clear from Fig. S3 that the transfer of the phenyl ring is unlikely to take place before the iodide is moved from the $\mathrm{Pd}$ to the $\mathrm{Zn}$ center. The latter process is practically barrier-less in electronic energy, while the former quickly surpasses $15 \mathrm{kcal} / \mathrm{mol}$. Thus, transferring the iodide ligand first, leads to a much more straight-forward, step-wise manner of reaching the TS structure.

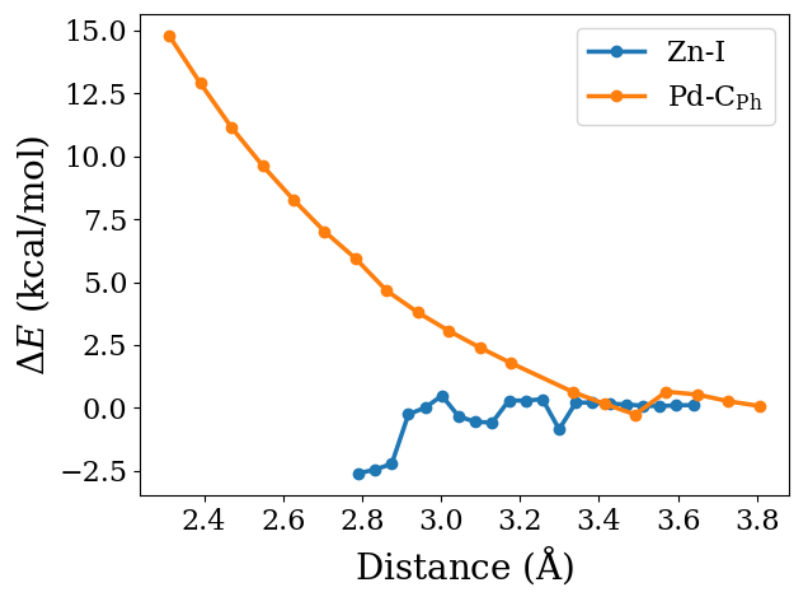

Figure S3: Relaxed potential energy surfaces for $\mathrm{Pd}-\mathrm{C}_{\mathrm{Ph}}$ and $\mathrm{Zn}-\mathrm{I}$ distances. The bonds were scanned independently of each other to compare the barriers. Note that electronic energies relative to the reactant contact pair (Ila) are displayed (cf. Scheme 3).
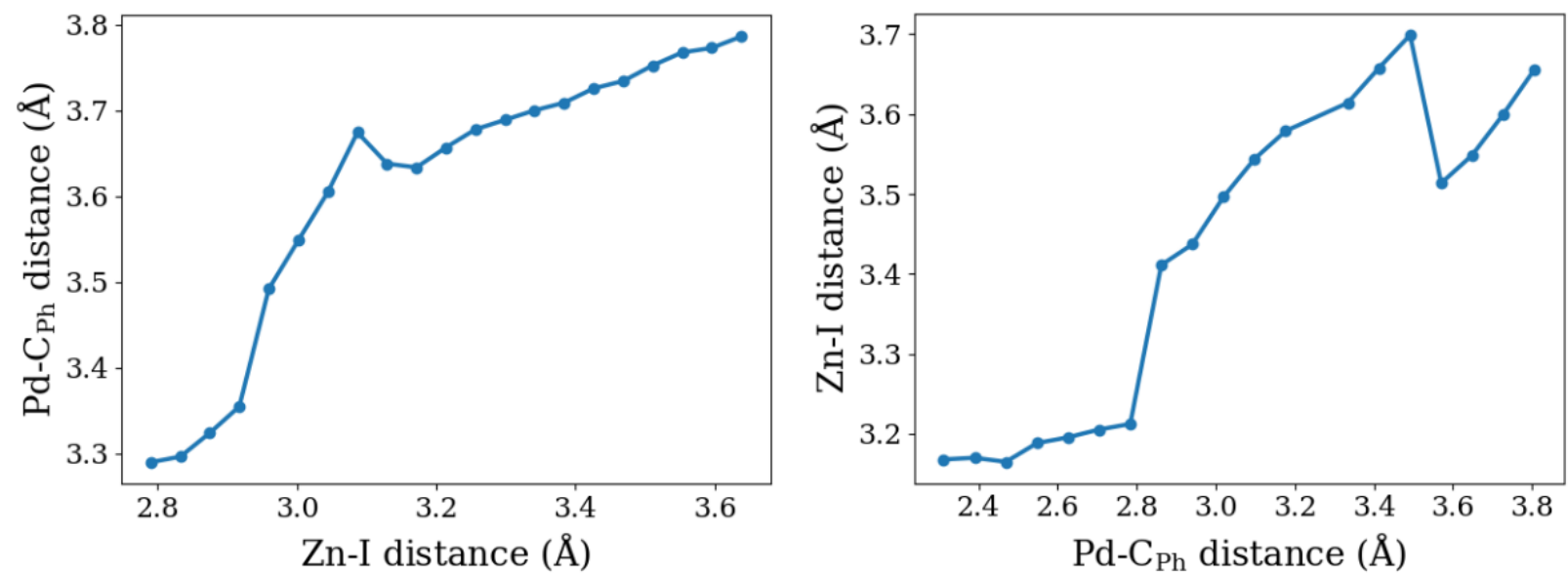

Figure S4: Coupling of the $\mathrm{Pd}-\mathrm{C}_{\mathrm{Ph}}$ and the $\mathrm{Zn}-\mathrm{I}$ distance. Both curves were obtained by respectively calculating a relaxed PES of the other one (cf. Fig. S3). 


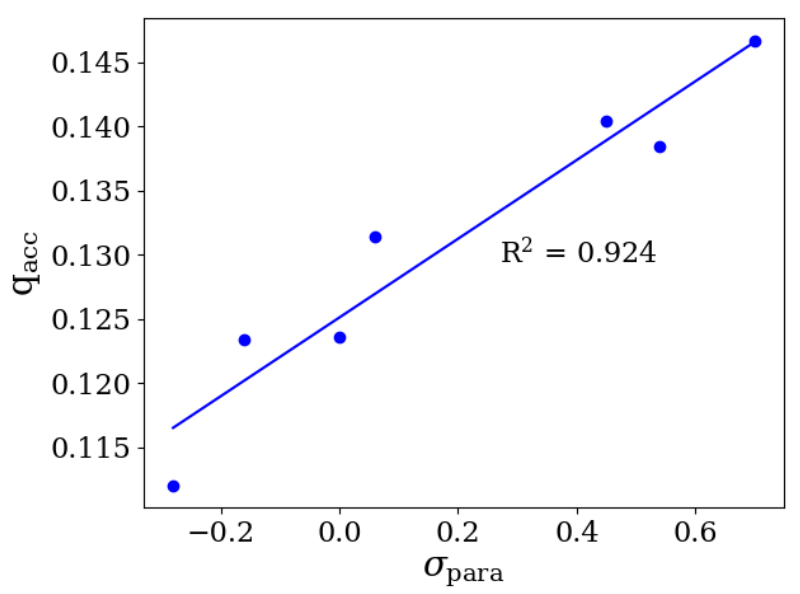

Figure S5: Relationship between the accumulated charge at the $\mathrm{Pd}$ center in the TS structure $q_{\text {acc }}$ and $\sigma_{\text {para }}$

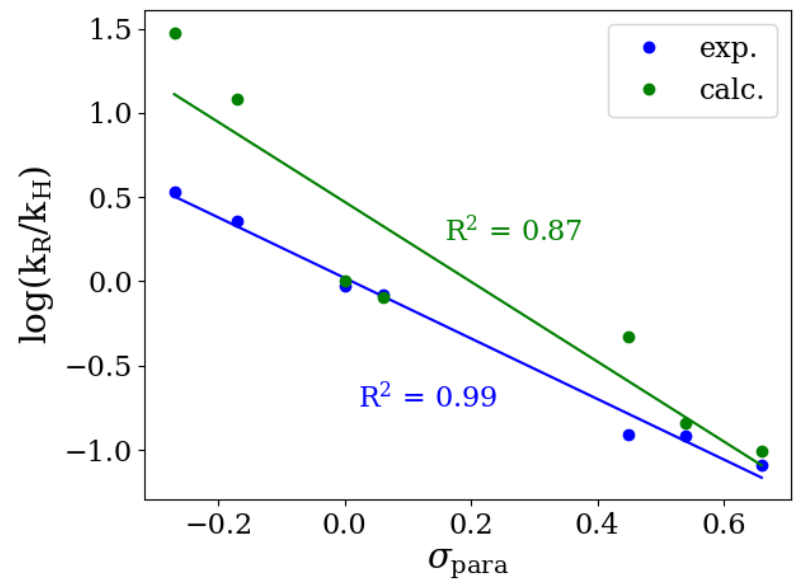

Figure S6: Relationship between $\log \left(\mathrm{k}_{\mathrm{R}} / \mathrm{k}_{\mathrm{H}}\right)$ from experiment and theory and $\sigma_{\text {para }}$. The experimental values are averaged for simpler comparison to the calculated ones. 


\section{References}

(1) Zalesskiy, S. S.; Ananikov, V. P. Organometallics 2012, 31, 2302-2309.

(2) Ball, N. D.; Gary, J. B.; Ye, Y.; Sanford, M. S. J. Am. Chem. Soc. 2011, 133, 7577-7584.

(3) Markies, B. A.; Canty, A. J.; de Graaf, W.; Boersma, J.; Janssen, M. D.; Hogerheide, M. P.; Smeets, W. J.; Spek, A. L.; van Koten, G. J. Organomet. Chem. 1994, 482, 191-199.

(4) Ball, N. D.; Kampf, J. W.; Sanford, M. S. J. Am. Chem. Soc. 2010, 132, 2878-2879.

(5) Hoare, J. L.; Lorenz, K.; Hovestad, N. J.; Smeets, W. J. J.; Spek, A. L.; Canty, A. J.; Frey, H.; van Koten, G. Organometallics 1997, 16, 4167-4173.

(6) Mann, G.; Baranano, D.; Hartwig, J. F.; Rheingold, A. L.; Guzei, I. A. J. Am. Chem. Soc. 1998, 120, $9205-9219$.

(7) Ogata, T.; Hartwig, J. F. J. Am. Chem. Soc. 2008, 130, 13848-13849. 